\title{
La integración de dispositivos móviles en el aula para la enseñanza del álgebra: el caso de la función lineal
}

\section{Using mobile devices in classroom for algebra teaching: the case of linear function} \begin{abstract}
Vladimir Ballesteros-Ballesteros
Carlos López-Torres
Marcela Torres-Rodríguez
Sébastien Lozano-Forero
Fundación Universitaria Los Libertadores, Bogotá D.C., Colombia
Resumen
Objetivo: Describir los efectos y las experiencias de la incorporación de la aplicación "Calculadora
Gráfica" de GeoGebra para el aprendizaje de la función lineal con estudiantes de undécimo grado a partir de la integración de dispositivos móviles al aula de clase. Método: Desde el paradigma pragmático, se empleó un diseño explicativo secuencial para orientar los esfuerzos investigativos conducentes a validar la hipótesis relacionada con una influencia positiva del aprovechamiento de teléfonos inteligentes y tabletas en el desempeño escolar. En la etapa cuantitativa se realizó un diseño experimental de cuatro grupos de Solomon y durante la etapa cualitativa se empleó un test actitudinal y se aplicaron entrevistas semiestructuradas con estudiantes que participaron del tratamiento con la aplicación móvil. Resultados: Los resultados obtenidos en el postest por los grupos experimentales fueron superiores a los obtenidos por los grupos control que recibieron una intervención mediada por recursos didácticos tradicionales y los estudiantes que recibieron el tratamiento manifestaron mayor interés y motivación por el aprendizaje del tema abordado. Discusión y Conclusiones: La integración de dispositivos móviles en el aula promueve otras formas innovadoras de aprender y desarrolla habilidades en los estudiantes, favoreciendo su motivación e interés.
\end{abstract}

Palabras clave: Aprendizaje móvil, función lineal, software de geometría dinámica, tecnologías digitales.

\begin{abstract}
Objective: To describe the effects and experiences of the incorporation of the GeoGebra "Graphing Calculator" app for linear function learning process with eleventh grade students based on the integration of mobile devices into the classroom. Method: From pragmatic paradigm, a sequential explanatory design was used to guide research efforts to judge the hypothesis related to a positive influence on the use of smartphones and tablets in school performance. In the quantitative stage, an experimental design of four Solomon groups was carried out, and during the qualitative stage, an attitudinal test and semi-structured interviews were applied with students who participated in the experiment with the above-mentioned application. Results: The results obtained in the posttest by the experimental groups were superior to those obtained by the control groups that received an intervention mediated by traditional didactic resources and the students who received the treatment expressed greater interest and motivation for the learning of the subject addressed. Discussion and Conclusions: The integration of mobile devices in the classroom promotes other innovative ways of learning and develops skills in students, favoring their motivation and interest.
\end{abstract}

Keywords: Mobile learning, linear function, dynamic geometry software, digital technologies.
Open Access:

ISSN: $0124-2121$

E-ISSN: $2665-2420$

Editor:

Dhayana Fernández Matos

TIPOLOGía DE ARTÍ́CULO
Copyright $\odot$

By Educación y Humanismo

Correspondencia: vladimir.ballesteros@libertadores.edu.co

Recibido: 02-08-2021 Aceptado: 08-11-2021 En línea desde: 11-01-2022

Cómo citar este artículo (APA): Ballesteros-Ballesteros, V., López-Torres, C., Torres-Rodríguez, M., \& Lozano-Forero, S. (2022). La integración de dispositivos móviles en el aula para la enseñanza del álgebra: el caso de la función lineal. Educación y Humanismo, 24(42), 1-20. https://doi.org/10.17081/eduhum.24.42.4044 


\section{Introducción}

Las últimas dos décadas han sido el escenario propicio para el desarrollo tecnológico creciente que ha permeado todos los aspectos de la vida del ser humano (Bauer, 2018), generando cambios en todos los ámbitos de la sociedad y permitiendo que muchas personas tengan acceso a la web, telefonía móvil, tabletas y comunicación global. Los jóvenes de hoy, que han nacido y crecido inmersos en la tecnología, desde el principio han contado con computadores, videojuegos, música digital y telefonía móvil; esto implica que piensen y procesen la información de manera diferente y sean reconocidos como nativos digitales, porque se han educado utilizando la lengua digital particular de Internet (Kesharwani, 2020); y este uso se ha convertido en un fenómeno internacional (Baron \& Campbell, 2012). Frente a esta situación, y a la manera como aprenden estas nuevas generaciones, las prácticas educativas se ven rezagadas y, por ello, es necesario integrar la tecnología al aula y avanzar hacia pedagogías [otras] centradas en el estudiante, utilizando eficientemente recursos TIC para mejorar el aprendizaje (Göksu \& Atici, 2013).

La literatura científica ha destacado los efectos positivos del uso de la tecnología en el aula de clase. A nivel escolar, se han reportado experiencias que reflejan los efectos positivos de la vinculación de recursos tecnológicos en el aprendizaje de las matemáticas (Zulnaidi, Oktavika, \& Hidayat, 2020). Antecede la implementación del software educativo Frizbi Mathematics 4, con una experiencia positiva de aprendizaje sobre multiplicación y división de números naturales y racionales (Pilli \& Aksu, 2013). También, el uso de Cabri Geometry como software útil para la enseñanza de la geometría (Bokosmaty, Mavilidi, \& Paas, 2017; Kösa \& Karakuş, 2010). Sin embargo, la tecnología digital que predomina en intervenciones e investigaciones en educación matemática es GeoGebra (İBİLİ, 2019); su versatilidad facilita la visualización de ideas matemáticas en niveles diferentes que van desde la educación primaria hasta la educación superior. Este software educativo, que se distingue por su facilidad de uso, reúne en un solo lugar geometría, álgebra y estadística, permitiendo manipular ecuaciones y, al tiempo, observar los cambios de sus gráficas, con la ventaja de poder particularizar un usuario para tener la posibilidad de guardar, exportar y trabajar en archivos propios, con un mecanismo de visualización, que alterna un sistema de álgebra computacional (SAC) con la variabilidad del Software de Geometría dinámica (SGD), permitiendo al usuario la interacción con distintas pantallas (Hohenwarter \& Jones, 2007). GeoGebra ha ganado popularidad en todo el mundo por su naturaleza libre, se ha traducido a más de 50 idiomas, se ha utilizado en 190 países y, como organización, apoya iniciativas e institutos para la investigación educativa.

Recientemente, se ha desarrollado una versión para dispositivos móviles con sistema operativo Android o iOS, respondiendo a las necesidades de transformación tecnológica de una sociedad móvil que requiere estar conectada, incrementar la movilidad física, interactiva e instantánea (Tomaschko \& Hohenwarter, 2018). Es necesario transformar la enseñanza 
que tiene lugar en el aula y trasladarla a un entorno que pueda tener lugar en la escuela, en el hogar o fuera de ellos (Bano, Zowghi, Kearney, Schuck, \& Aubusson, 2018), promoviendo un aprendizaje actual y perspectivo, personalizado, contextualizado y no restringido a espacio ni tiempo. Así, y en respuesta a las nuevas necesidades de formación, surge una nueva tendencia educativa, con gran potencial, en el escenario de la innovación educativa denominada mobile learning (aprendizaje móvil) que, aunque joven, ha evolucionado rápidamente y proporciona una experiencia de aprendizaje diferente a la determinada por las tecnologías de aprendizaje electrónico convencional donde los estudiantes aún se encuentran anclados a un solo lugar (Pachler, Bachmair, \& Cook, 2010a).

Con este escenario renovado y con pleno reconocimiento de las necesidades de aprendizaje de los estudiantes del Colegio Alfredo Iriarte (IED), se propuso la siguiente pregunta de investigación: ¿Qué influencia tiene en el desempeño académico y el desarrollo actitudinal de los estudiantes el uso de la aplicación móvil "¿Calculadora Gráfica" de GeoGebra (en adelante ACGG) para el aprendizaje de la función lineal? Para responder a esta pregunta, se trazó como objetivo general la necesidad de describir y analizar los efectos y las experiencias de la incorporación de la ACGG para el aprendizaje de la función lineal con estudiantes de undécimo grado a partir de la integración de dispositivos móviles al aula de clase $y$, de este modo, validar la hipótesis asociada a una influencia positiva del uso de esta aplicación en el aprendizaje de este tópico.

\section{Revisión de la literatura}

\section{Aprendizaje Móvil o m-learning}

El aprendizaje móvil (m-learning) consiste en un modelo de aprendizaje que le permite al estudiante acceder a información y material educativo en cualquier momento y lugar, haciendo uso de dispositivos de mano inalámbricos como teléfonos móviles inteligentes, tabletas y computadores portátiles (Pachler, Bachmair, \& Cook, 2010b). A favor de este modelo, se encuentra el uso en cualquier momento y lugar, la facilidad de acceder a este tipo de dispositivos en términos de precio y tamaño, y su soporte en tecnologías modernas populares para los estudiantes. Otras ventajas se relacionan con la personalización, estar centrado en el usuario, la movilidad, el desarrollo en red y la ubicuidad, permitiendo que las personas puedan obtener y compartir información cuando lo necesiten (Motiwalla, 2007).

Los avances tecnológicos actuales han generado nuevos retos para la educación matemática (Zazkis, 2020). Se ha demostrado, a partir de distintas publicaciones, que el uso de las TIC en la educación tiene efectos positivos en los procesos educativos (Cox \& Marshall, 2007; Huang \& Price, 2016). Específicamente, ha surgido un interés particular por el aprendizaje a partir de la integración de dispositivos móviles ya que se consideran como una herramienta de fácil acceso (Vanden Abeele, 2016). Por ejemplo, se ha demostrado que 
los encuentros asincrónicos, usando dispositivos móviles, influyen de manera positiva en el rendimiento de los estudiantes, puesto que este tipo de actividades permite reflexionar, discutir y compartir más información que promueva la construcción de conocimiento (Lan, Tsai, Yang \& Hung, 2012). También, se ha señalado que el acceso a los dispositivos móviles aumenta la frecuencia en el acceso a los recursos digitales y, esto, transforma de manera positiva las prácticas educativas (Heflin, Shewmaker \& Nguyen, 2017). Así, en la literatura científica se han registrado efectos positivos de la implementación del aprendizaje móvil en el aprendizaje de idiomas (Azar \& Nasiri, 2014; Burston, 2011; Huang, Yang, Chiang \& Su, 2016; Jeon, 2015), en la enseñanza del arte (Schuck, Kearney \& Burden, 2017; Wu et al., 2012), en el ámbito de la enseñanza de la historia (Cruz, Carvalho \& Araújo, 2017; Harley, Poitras, Jarrell, Duffy \& Lajoie, 2016; König \& Bernsen, 2014), entre otros.

\section{GeoGebra}

Alrededor del mundo se han implementado varios recursos digitales para facilitar los procesos de aprendizaje y enseñanza de las matemáticas con mayor énfasis en GeoGebra. Sobre éste, se han llevado a cabo varias investigaciones que arrojan resultados positivos en su implementación. Dichos resultados apuntan a consolidar GeoGebra como una herramienta que permite construir objetos geométricos y comprender sus conceptos asociados sin tener conocimientos avanzados en programación (Pamungkas, Rahmawati \& Dinara, 2020), aprender probabilidad (Aizikovitsh-Udi \& Radakovic, 2012; Chan \& Ismail, 2014) y enseñar funciones complejas a través de técnicas de coloración de dominios (Breda \& Santos, 2016); otros resultados importantes, se relacionan con el aprendizaje de números enteros (Reisa, 2010), del concepto de elipse (Ljajko \& Ibro, 2013), de probabilidad condicional y teorema de Bayes (Radakovic \& McDougall, 2012), de teoremas del cálculo diferencial (Caligaris, Schivo \& Romiti, 2015; Diković, 2009), en el aprendizaje de la trigonometría (Zengin, Furkan, \& Kutluca, 2012) y en el aprendizaje de la física (Koláŕ, 2019).

\section{Método}

\section{Diseño de investigación y muestra}

El diseño de investigación utilizado en la presente investigación fue el explicativo secuencial, caracterizado por dos etapas: en la primera se recolectan y analizan datos cuantitativos y, en la segunda, se valoran datos cualitativos; finalmente la interpretación de los hallazgos de ambas etapas permiten la extracción de conclusiones (Ivankova, Creswell, \& Stick, 2006). En la etapa cuantitativa, se tomaron cuatro grupos: dos grupos experimentales y dos grupos control, para garantizar la validez externa, atendiendo al planteamiento de Solomon (Solomon, 1949), quien afirmó que la prueba previa (pretest) 
puede influir en la efectividad del método de enseñanza. Los dos grupos, denominados experimentales, contaron con un total de sesenta y seis (66) estudiantes y los otros dos grupos, denominados control, con setenta (70) estudiantes pertenecientes a undécimo grado del Colegio Alfredo Iriarte IED, ubicado al sur de Bogotá, D.C. (Tabla 1). Posteriormente, en la etapa cualitativa, se aplicó un test actitudinal a los dos grupos experimentales y, posteriormente, se realizaron entrevistas semiestructuradas a diez (10) estudiantes de esos grupos para determinar su percepción frente al uso de la ACGG en el aprendizaje de la función lineal.

Las intervenciones se desarrollaron a través de dos unidades didácticas; la primera, el tratamiento experimental, integró la ACGG para el desarrollo de las actividades planeadas y, la otra, integró recursos didácticos tradicionales para el mismo propósito. Los cuatro grupos abordaron ejercicios y problemas idénticos en cantidad y nivel de dificultad que fueron preparados por los investigadores y validados por expertos en dos fases.

Tabla 1.

Diseño del experimento

\begin{tabular}{r|c|c|c|c|} 
& $\begin{array}{c}\text { Grupo } \\
\text { Experimental I } \\
\left(\mathrm{GE}_{1}\right)\end{array}$ & $\begin{array}{c}\text { Grupo } \\
\text { Experimental II } \\
\left(\mathrm{GE}_{2}\right)\end{array}$ & $\begin{array}{c}\text { Grupo Control I } \\
\left(\mathrm{GC}_{1}\right)\end{array}$ & $\begin{array}{c}\text { Grupo Control II } \\
\left(\mathrm{GC}_{2}\right)\end{array}$ \\
\hline Pretest & Sí & No & Sí & No \\
\hline Tratamiento & Sí & Sí & No & No \\
\hline Postest & Sí & Sí & Sí & Sí \\
\cline { 2 - 5 } & & &
\end{tabular}

Fuente: elaboración propia (2019)

\section{Procedimiento}

Se inició con la aplicación de un pretest, con validación de expertos en dos fases, que incluyó diez preguntas a dos grupos elegidos aleatoriamente: un grupo experimental $\left(\mathrm{GE}_{1}\right)$ y uno control $\left(\mathrm{GC}_{1}\right)$. Este pretest indagaba por el manejo y comprensión que los estudiantes tenían de la función lineal. Posteriormente, se adelantó el despliegue de la unidad didáctica con los dos grupos experimentales, en tres bloques temáticos, a lo largo de dos sesiones, cada una con una duración aproximada de ciento diez minutos. En el primer bloque temático, se hizo una ambientación del uso de la tecnología en el aula, una introducción a la ACGG y a las nociones básicas de función, así como a las cuatro formas diferentes de representarlas. En el segundo bloque temático se trabajó alrededor de los elementos que componen la función lineal: pendiente y punto de corte. En el tercer bloque temático se modelaron diferentes situaciones a través de la función lineal. En el caso de los grupos control se utilizó un despliegue similar, pero con recursos tradicionales como calculadora científica, regla, lápiz y papel. Al finalizar la intervención, y en sintonía con el diseño de investigación seleccionado, se hizo la recolección de datos y el análisis cuantitativo correspondiente con los resultados del postest que se aplicó a los cuatro grupos; 
posteriormente, se evaluaron los datos cualitativos obtenidos de la encuesta actitudinal y las entrevistas semiestructuradas a los estudiantes frente al manejo y uso de la ACGG.

\section{Datos Cuantitativos}

Se realizó un análisis de varianza (ANOVA) de dos factores (cada factor puede entenderse como una fuente de variabilidad, en este caso, la implementación del pretest y la efectividad de los tratamientos); se verificó la existencia de evidencia que juzgara la hipótesis de interacciones entre las dos variables en estudio para cotejar el efecto de cada una de estas variables junto con la posible interacción en el resultado del postest (Braver \& Braver, 1988). Esta estrategia de análisis ha sido ampliamente implementada en educación médica (Cheng Lim et al., 2019), aprendizaje basado en videojuegos (All, Plovie, Castellar, \& Van Looy, 2017), desarrollo infantil (Rybanska, McKay, Jong \& Whitehouse, 2018), salud pública (Van Landschoot, Portzky \& Van Heeringen, 2017), entre otros. Para el procesamiento de estos datos, se utilizó el software estadístico $\mathrm{R}$, en su versión de escritorio 1.2.1335, y se fijó el nivel de significancia en $5 \%$ (es decir, se rechazará una hipótesis planteada si el p-valor asociado es menor a 0.05). Con estas condiciones iniciales establecidas, se buscó juzgar la hipótesis general "el uso de la ACGG influye de manera positiva en el aprendizaje de la función lineal".

\section{Datos Cualitativos}

Para obtener información acerca del uso y percepción que los estudiantes tuvieron de la experiencia de aprendizaje, se realizó una encuesta actitudinal fundamentada en el Modelo de Motivación ARCS-V (Keller, 2016) y, a continuación, entrevistas semiestructuradas con un grupo de estudiantes seleccionados aleatoriamente. El modelo ARCS-V se fundamenta en cinco categorías fundamentales: Atención (relacionada con la curiosidad y el interés), Relevancia (en relación con la percepción de los estudiantes sobre la correspondencia entre las prácticas educativas y sus estilos de aprendizaje), Confianza (relacionada con las experiencias de éxito), Satisfacción (asociada con la mezcla apropiada de resultados intrínsecamente y extrínsecamente gratificantes que sustentan los comportamientos de aprendizaje deseables y desalientan los no deseados) y, finalmente, la Volición (asociada con la capacidad de persistir en el aprendizaje). El instrumento se constituyó a partir de diez ítems con validación en dos fases. Se asociaron dos preguntas con cada una de las categorías del modelo ARCS-V como se muestra a continuación:

Tabla 2.

Configuración del instrumento de evaluación

Categoría Ítem

\begin{tabular}{cc}
\hline Atención & P6 y P10 \\
\hline Relevancia & P3 y P4 \\
\hline Confianza & P5 y P7 \\
\hline
\end{tabular}




\begin{tabular}{cc}
\hline Satisfacción & P1 y P2 \\
\hline Volición & P8 y P9 \\
\hline
\end{tabular}

Fuente: elaboración propia (2019)

Posteriormente, se desarrollaron entrevistas semiestructuradas con un grupo de diez estudiantes, en un ambiente distinto al aula de clase, para indagar respecto a su percepción frente a la implementación de la ACGG para el aprendizaje de la función lineal.

\section{Resultados}

Una vez recopilada la evidencia empírica, se procedió con el análisis descriptivo. De este modo, en la figura 1 , se ilustran los valores promedio de los resultados obtenidos por los cuatro grupos en el postest, considerando la presencia del pretest y el tratamiento realizado. A partir de los resultados obtenidos, es posible conjeturar que la intervención tuvo un efecto positivo en el desempeño de los estudiantes de los grupos experimentales frente a los resultados de los grupos control.

\section{Figura 1.}

Promedio de los resultados de los cuatro grupos



Fuente: elaboración propia (2019) 
Figura 2.

Distribución de resultados de los cuatro grupos

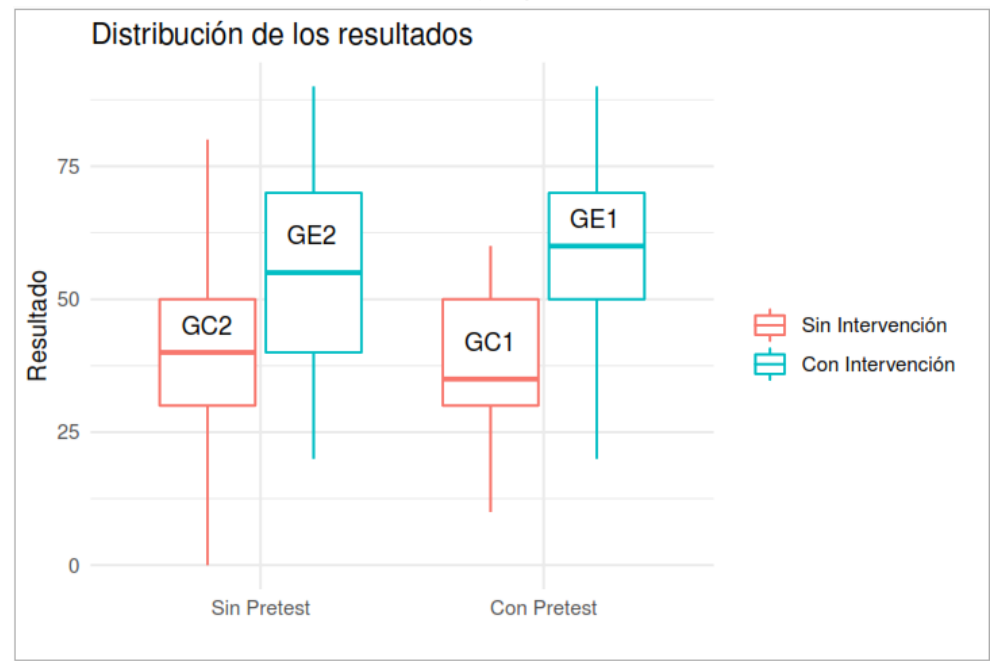

Fuente: elaboración propia (2019)

En la figura 2, se presenta la distribución de los resultados obtenidos en el postest por parte de los cuatro grupos de estudiantes. Se puede apreciar que el grupo experimental 1 $\left(\mathrm{GE}_{1}\right)$, que presentó pretest y además recibió tratamiento con la ACGG, mostró un mejor desempeño que los demás grupos. También, se debe destacar que el rendimiento del Grupo Experimental 2 ( $\mathrm{GE}_{2}$, que no recibió pretest) es superior al desempeño de los grupos control. Por otra parte, se detectaron los siguientes sucesos: i) el Grupo Experimental $2\left(\mathrm{GE}_{2}\right)$ presenta mayor variabilidad intercuartílica entre los grupos considerados y ii) el Grupo Control $1\left(\mathrm{GC}_{1}\right)$ tiene una distribución asimétrica a la izquierda.

Como se señaló anteriormente, con el ánimo de encontrar evidencia estadísticamente significativa que respalde la hipótesis sobre la influencia positiva en el aprendizaje de la función lineal cuando existe la mediación de la ACGG, en la tabla 3 se presentan los resultados de la ANOVA de dos vías (o factores) con interacción. El p-valor calculado para el parámetro asociado a la interacción es de 0.09538, que se constituye como evidencia estadísticamente significativa para concluir, con el $5 \%$ de significancia, que no hay interacciones significativas. Es decir, no existe un efecto potenciador en el resultado del postest asociado a alguna combinación particular entre pretest e intervención.

\section{Tabla 3.}

Reporte ANOVA con interacciones

\begin{tabular}{ccccc}
\hline Parámetro & Estimación & Error Estándar & Valor F & p-valor \\
\hline Intercepto & 62836 & 1 & 199,6230 & $<2$ e -16 \\
\hline Pretest & 624 & 1 & 1,9833 & 0,16161 \\
\hline Intervención & 2029 & 1 & 6,4466 & 0,01238 \\
\hline Pretest: & 889 & 1 & 2,8251 & 0,09538 \\
\hline
\end{tabular}


Intervención

Residuales 38088 121

Fuente: elaboración propia (2019)

Una vez establecida la no significancia estadística de la interacción, se procede a validar los efectos individuales de las variables (pretest e intervención) a partir de una ANOVA de dos factores sin interacción. Los resultados se encuentran en la tabla 4, evidenciando los pvalores 0.7544 y $7.88 \mathrm{e}-07$ en las pruebas de significancia para los parámetros asociados a las variables pretest e intervención respectivamente. Es decir, con una significancia del $5 \%$, hay evidencia estadísticamente significativa para concluir que el pretest no representó una fuente de variabilidad relevante para el resultado obtenido en el postest. Por otro lado, con la misma significancia, hay evidencia estadísticamente significativa para concluir que la intervención basada en el uso de la ACGG es relevante para el resultado obtenido en el postest. Esto es, es posible concluir que el uso de la ACGG influye de manera positiva en el aprendizaje de la función lineal en los estudiantes de los grupos experimentales.

Tabla 4.

Reporte ANOVA sin interacciones

\begin{tabular}{lcccc}
\hline \multicolumn{1}{c}{ Parámetro } & Estimación & Error Estándar & Valor $\mathbf{F}$ & p-valor \\
\hline Intercepto & 73041 & 1 & 228,6209 & 2,2 e -16 \\
\hline Pretest & 31 & 1 & 0,0983 & 0,7544 \\
\hline Intervención & 8663 & 1 & 27,1142 & 7,88 e -07 \\
\hline Residuales & 38977 & 122 & & \\
\hline
\end{tabular}

Fuente: elaboración propia (2019)

Para tener certeza sobre la conveniencia del modelo presentado en la tabla 3, se procede con su validación. Para esto se verifican los dos supuestos fundamentales realizados sobre el modelo: (a) Normalidad en los residuos del modelo y (b) Homogeneidad en las varianzas de la variable respuesta para las diferentes combinaciones de valores de las variables independientes. Para la validación de (a), se procede a realizar la prueba de hipótesis de normalidad de Jarque-Bera en los residuos $\left(H_{0}\right.$ : Los datos provienen de una distribución normal versus $H_{1}$ : Los datos no provienen de una distribución normal). La misma, tiene un p-valor de 0.5366, por lo que hay evidencia estadísticamente significativa para afirmar que los datos siguen una distribución normal. En la misma línea, la figura 3 muestra el gráfico cuantil-cuantil de los residuos del modelo, dando cuenta que los cuantiles empíricos tienen concordancia con los cuantiles teóricos de una distribución normal. 


\section{Figura 3.}

Cuantil-cuantil de los residuos del modelo propuesto



Fuente: elaboración propia (2019)

Finalmente, evidenciar la homogeneidad de las varianzas en los grupos garantiza que la variable de interés (resultados en el postest) tenga una varianza estable a lo largo de las diferentes combinaciones de las categorías de las variables explicativas (resultado en el postest y tipo de intervención). En congruencia con lo anterior, se debe verificar el supuesto de homocedasticidad. En la figura 4 se muestra que no existe ningún patrón destacado en la línea de tendencia desplegada, puesto que el intervalo de confianza mostrado contiene al cero.

\section{Figura 4.}

Cuantil-cuantil de los residuos del modelo propuesto



Fuente: elaboración propia (2019) 
Por otra parte, en la figura 5 se presenta el análisis de la encuesta actitudinal que se aplicó a los sesenta y seis estudiantes de los grupos experimentales, donde se puede evidenciar una aceptación importante de la ACGG como recurso didáctico para el aprendizaje de la función lineal:

Figura 5.

Resultados de la encuesta actitudinal
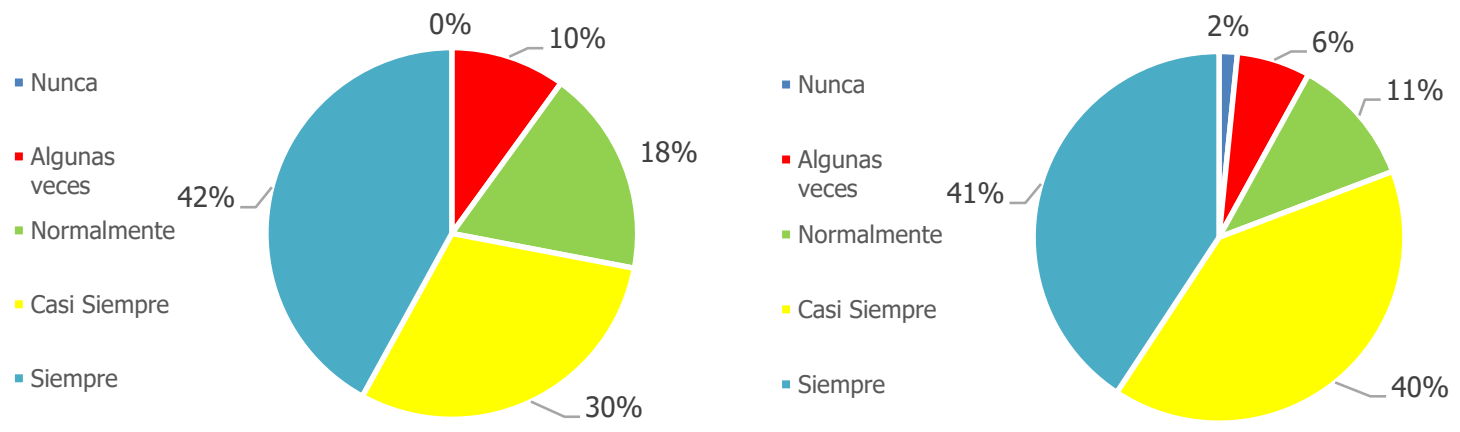

Ítem 1. Para mí es fácil usar ACGG: La mayor cantidad de observaciones en este ítem se concentró en la categoría "Siempre".

Ítem 2. Me gusta utilizar $A C G G$ en clase de matemáticas: La mayor cantidad de observaciones en este ítem se concentró en la categoría "Siempre".


Ítem 3. Tener la aplicación de ACGG en mi celular o tableta me permite aprender en cualquier momento y cualquier lugar: La mayor cantidad de observaciones en este ítem se concentró en la categoría "Casi siempre".

Ítem 4. $L a A C G G$ me permite relacionar diferentes representaciones de la función: La mayor cantidad de observaciones en este ítem se concentró en la categoría "Siempre". 




Ítem 5. $L a A C G G$ me ayuda a aprender conceptos matemáticos: La mayor cantidad de observaciones en este ítem se concentró en la categoría "Casi siempre".

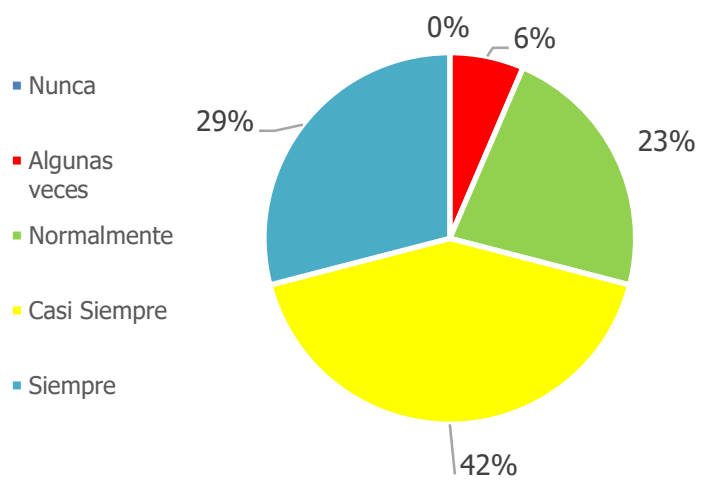

Ítem 7. Al utilizar celular o tableta mejoro mi comprensión de los temas de matemáticas: La mayor cantidad de observaciones en este ítem se concentró en la categoría "Casi siempre".

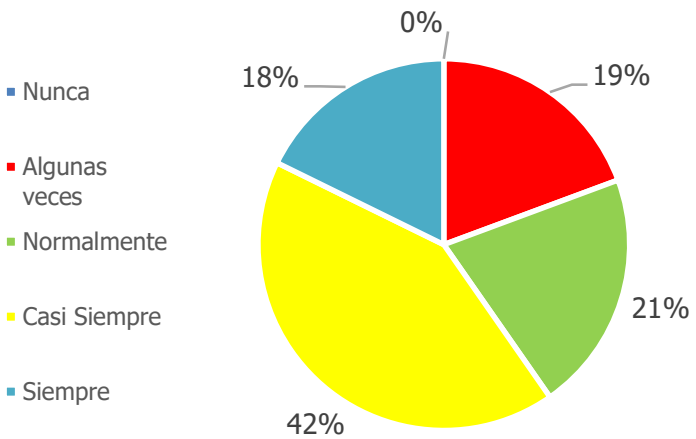

Ítem 9. Es importante el uso del celular o tableta en las clases: La mayor cantidad de observaciones en

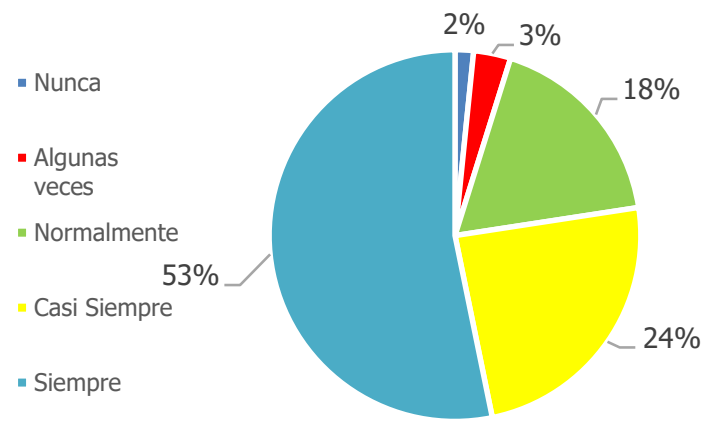

Ítem 6. Me gusta que se use el celular o la tableta en las clases de matemáticas: La mayor cantidad de observaciones en este ítem se concentró en la categoría "Siempre".

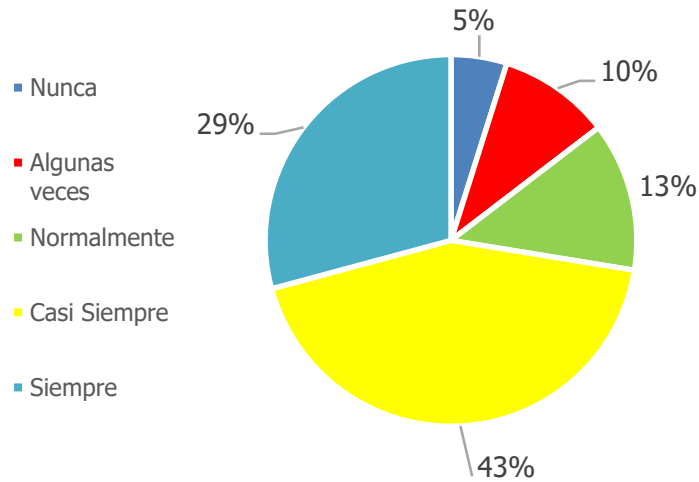

Ítem 8. Las aplicaciones que se descargan en el celular o tableta contribuyen a mejorar mi desempeño académico: La mayor cantidad de observaciones en este ítem se concentró en la categoría "Casi siempre".

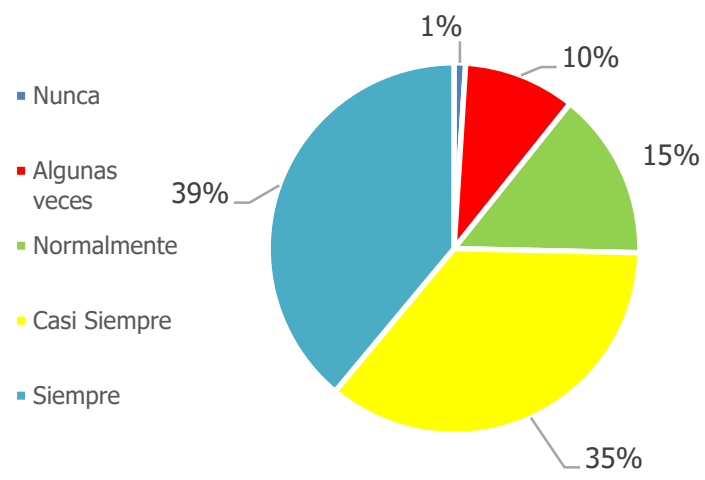

Ítem 10. El trabajo con la ACGG me motiva a trabajar y participar en la clase de matemáticas: La 
este ítem se concentró en la categoría "Casi siempre". mayor cantidad de observaciones en este ítem se concentró en la categoría "Siempre".

Fuente: elaboración propia (2019)

Posteriormente, se realizaron las entrevistas semiestructuradas a diez estudiantes de los grupos experimentales que fueron elegidos aleatoriamente. A continuación, se presentan algunas percepciones de los estudiantes entrevistados frente a la incorporación de la ACGG en la clase de matemáticas:

\section{Figura 6.}

Percepción del estudiante E03 frente a la ACGG

ts una aplicacion para el aprendiacie y descirrollo der gada nos facilita y hos a graficación en el plano

Fuente: elaboración propia (2019)

\section{Figura 7.}

Percepción del estudiante E04 frente a la ACGG

me Dorcee Divortido Porfac mrontros a ec Aprendemos Puedemo interactuar con piversos elomentos de Geo Gebra 7 A la vez es mos facrl a prender.

Fuente: elaboración propia (2019)

\section{Figura 8.}

Percepción del estudiante EO7 frente a la ACGG

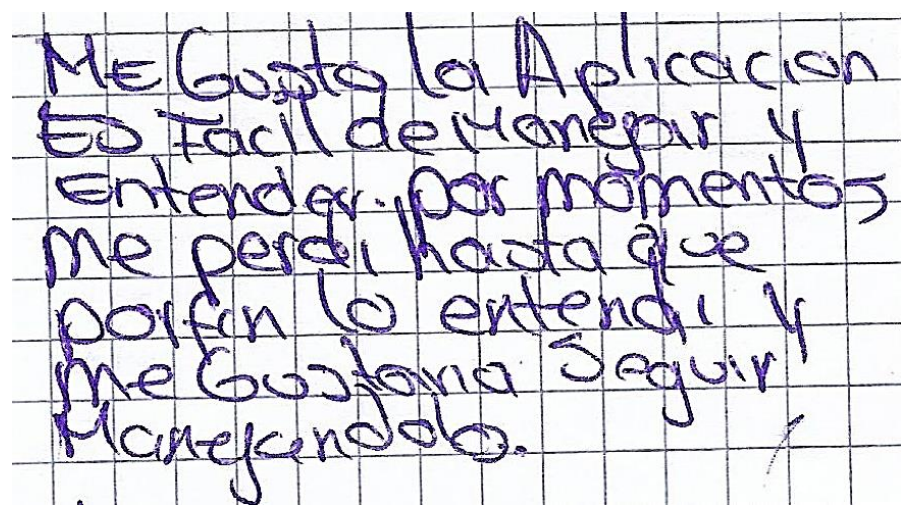

Fuente: elaboración propia (2019) 
Figura 9.

Percepción del estudiante E09 frente a la ACGG



Fuente: elaboración propia (2019)

\section{Discusiones y conclusiones}

La educación matemática enfrenta nuevos retos y desafíos; uno de ellos es involucrar la tecnología en el aula. Es así como ha surgido el aprendizaje móvil en respuesta a la necesidad de involucrar dispositivos móviles en los procesos de aprendizaje y enseñanza. Este estudio plantea que el aprendizaje móvil es una opción adecuada y efectiva para los jóvenes de hoy, debido a que promueve mayor compromiso académico al involucrar los dispositivos móviles que utilizan a diario y con los cuales se encuentran familiarizados. Este tipo de aprendizaje ofrece nuevas oportunidades; en el caso de este proyecto de investigación, proporcionó la oportunidad de abordar la función lineal de una manera diferente a la tradicional a través de la ACGG.

Esta aplicación móvil permitió un cambio en la clase porque además de ser un método innovador, es de libre uso y descarga en dispositivos móviles, por lo que no se requiere conexión a internet para ser usada, permitiendo que los estudiantes la usaran en cualquier momento en el aula de clase y también fuera de ella, facilitando la práctica educativa en congruencia con los planteamientos de Pimmer, Mateescu \& Gröhbiel (2016). También se evidenció que el uso de la ACGG incidió de manera positiva en el rendimiento del postest en los grupos experimentales, que superó en un $17 \%$ al desempeño de los grupos control. Esta aplicación para dispositivos móviles, se consolida como una valiosa herramienta tecnológica para potenciar el aprendizaje ya que, además de superar la barrera de la falta de conexión a Internet, contribuye a romper las barreras de tiempo y lugar.

La ACGG influye positivamente en el proceso de aprendizaje de la función lineal, ya que permite a los estudiantes hacer uso de los deslizadores y visualizar los cambios que tiene la representación gráfica de la función lineal $y$, simultáneamente, poder modificar la representación algebraica y la representación numérica. De esta manera la ACGG favoreció la construcción paulatina de los conceptos de punto de corte y pendiente; esto se encuentra en concordancia con el planteamiento hecho por Hohenwarter y Jones (2007) cuando 
afirman que esta herramienta permite la visualización de las ideas matemáticas en cualquier nivel. También, y en congruencia con Drijvers (2015), la ACGG permite que los estudiantes sientan que están trabajando con algo que tiene una existencia palpable. Los estudiantes involucrados en la intervención, tuvieron la oportunidad de trabajar con una rica diversidad de representaciones del concepto. $Y$ en cuanto a la modelación de situación la ACGG contribuye, en ahorro de tiempo y visualización, a la resolución de los diferentes problemas planteados.

Los resultados de este proyecto de investigación coinciden con los resultados de Hwang \& Chang (2011), en el sentido que el uso de este recurso digital promueve el interés y la actitud de aprendizaje de los estudiantes en la clase de matemáticas. También, se debe señalar que la incorporación de dispositivos móviles en el aula propicia nuevos ambientes de aprendizaje que, en este caso, favoreció el aprendizaje de la función lineal en concordancia con los estudios hechos en México por Benítez Armas (2016) y en Sudáfrica por Mudaly \& Fletcher (2019). Esta aplicación móvil sirve de apoyo en el aprendizaje de funciones, optimiza el tiempo y ayuda a visualizar las diferentes representaciones. Su interfaz gráfica permite al estudiante hacer una mejor asociación entre las representaciones visual, algebraica y numérica, promoviendo un mejor desempeño en la prueba estandarizada de salida. A partir de la integración de dispositivos móviles, los resultados planteados aquí están en la misma línea argumentativa que los planteamientos de Sung, Chang \& Liu (2016), respecto a que la tecnología móvil refuerza métodos educativos innovadores y desarrolla habilidades en los estudiantes, favoreciendo su motivación e interés.

\section{Referencias}

Aizikovitsh-Udi, E., \& Radakovic, N. (2012). Teaching probability by using geogebra dynamic tool and implemanting critical thinking skills. Procedia-Social and Behavioral Sciences, (46), 4943-4947. https://doi.org/10.1016/j.sbspro.2012.06.364

All, A., Plovie, B., Castellar, E. P. N., \& Van Looy, J. (2017). Pre-test influences on the effectiveness of digital-game based learning: A case study of a fire safety game. $\begin{array}{llll}\text { Computers } \& \text { Education, 24-37. } & \text { (114), }\end{array}$ https://doi.org/10.1016/j.compedu.2017.05.018

Azar, A. S., \& Nasiri, H. (2014). Learners' attitudes toward the effectiveness of mobile assisted language learning (MALL) in L2 listening comprehension. Procedia-Social and Behavioral Sciences, (98), 1836-1843. https://doi.org/10.1016/j.sbspro.2014.03.613

Bano, M., Zowghi, D., Kearney, M., Schuck, S., \& Aubusson, P. (2018). Mobile learning for 
science and mathematics school education: A systematic review of empirical evidence. Computers \& Education, (121), 30-58. https://doi.org/10.1016/j.compedu.2018.02.006

Baron, N. S., \& Campbell, E. M. (2012). Gender and mobile phones in cross-national context. Language Sciences, 34(1), 13-27. https://doi.org/10.1016/j.langsci.2011.06.018

Bauer, L. B. (2018). A necessary addiction: Student conceptualizations of technology and its impact on teaching and learning. Journal of College Reading and Learning, 48(1), 67-81. https://doi.org/10.1080/10790195.2017.1365668

Benítez Armas, A. F. (2016). Un experimento con GeoGebra (app) para el desarrollo del pensamiento matemático. Revista de Arte y Humanidades, 3(4), 9-22. Retrieved from https://www.upaep.mx/images/revista_artes_humanidades/pdf/AH_4212.pdf

Bokosmaty, S., Mavilidi, M.-F., \& Paas, F. (2017). Making versus observing manipulations of geometric properties of triangles to learn geometry using dynamic geometry software. Computers \& Education, (113), 313-326. https://doi.org/10.1016/j.compedu.2017.06.008

Braver, M. W., \& Braver, S. L. (1988). Statistical treatment of the Solomon four-group design: A meta-analytic approach. Psychological Bulletin, 104(1), 150. https://doi.org/10.1037/0033-2909.104.1.150

Breda, A. M. D., \& Santos, J. M. D. S. Dos. (2016). Complex functions with GeoGebra. Teaching Mathematics and Its Applications: An International Journal of the IMA, 35(2), 102-110. https://doi.org/10.1093/teamat/hrw010

Burston, J. (2011). Realizing the potential of mobile phone technology for language learning. IALLT Journal of Language Learning Technologies, 41(2), 56-71. https://doi.org/10.17161/iallt.v41i2.8490

Caligaris, M. G., Schivo, M. E., \& Romiti, M. R. (2015). Calculus \& GeoGebra, an interesting partnership. Procedia-Social and Behavioral Sciences, (174), 1183-1188. https://doi.org/10.1016/j.sbspro.2015.01.735

Chan, S. W., \& Ismail, Z. (2014). Developing statistical reasoning assessment instrument for high school students in descriptive statistics. Procedia-Social and Behavioral Sciences, (116), 4338-4343. https://doi.org/10.1016/j.sbspro.2014.01.943

Cheng Lim, S., Mustapha, F. I., Aagaard-Hansen, J., Calopietro, M., Aris, T., \& BjerreChristensen, U. (2019). Impact of Continuing Medical Education for Primary Healthcare Providers in Malaysia on Diabetes Knowledge, Attitudes, Skills and Clinical Practices. Medical Education Online, (25), 1-11. 
https://doi.org/10.1080/10872981.2019.1710330

Cox, M. J., \& Marshall, G. (2007). Effects of ICT: do we know what we should know? Education and Information Technologies, 12(2), 59-70. https://doi.org/10.1007/s10639-007-9032-x

Cruz, S., Carvalho, A. A. A., \& Araújo, I. (2017). A game for learning history on mobile devices. Education and Information Technologies, 22(2), 515-531. https://doi.org/10.1007/s10639-016-9491-z

Diković, L. (2009). Applications GeoGebra into teaching some topics of mathematics at the college level. Computer Science and Information Systems, 6(2), 191-203. https://doi.org/10.2298/CSIS0902191D

Drijvers, P. (2015). Digital technology in mathematics education: Why it works (or doesn't). Selected Regular Lectures from the 12th International Congress on Mathematical Education, 135-151. https://doi.org/10.1007/978-3-319-17187-6_8

Göksu, İ., \& Atici, B. (2013). Need for mobile learning: technologies and opportunities. Procedia-Social and Behavioral Sciences, 103, 685-694. https://doi.org/10.1016/j.sbspro.2013.10.388

Harley, J. M., Poitras, E. G., Jarrell, A., Duffy, M. C., \& Lajoie, S. P. (2016). Comparing virtual and location-based augmented reality mobile learning: emotions and learning outcomes. Educational Technology Research and Development, 64(3), 359-388. https://doi.org/10.1007/s11423-015-9420-7

Heflin, H., Shewmaker, J., \& Nguyen, J. (2017). Impact of mobile technology on student attitudes, engagement, and learning. Computers \& Education, (107), 91-99. https://doi.org/10.1016/j.compedu.2017.01.006

Hohenwarter, M., \& Jones, K. (2007). Ways of linking geometry and algebra, the case of Geogebra. Proceedings of the British Society for Research into Learning Mathematics, 27(3), 126-131. http://eprints.soton.ac.uk/id/eprint/50742

Huang, C. S. J., Yang, S. J. H., Chiang, T. H. C., \& Su, A. Y. S. (2016). Effects of situated mobile learning approach on learning motivation and performance of EFL students. Journal of Educational Technology \& Society, 19(1), 263-276. https://doi.org/https://www.jstor.org/stable/jeductechsoci.19.1.263

Huang, R., \& Price, J. K. (2016). ICT in education in global context. Springer, (10), 973978. https://doi.org/10.1007/978-3-319-19234-5_5

Hwang, G.-J., \& Chang, H.-F. (2011). A formative assessment-based mobile learning approach to improving the learning attitudes and achievements of students. 
Computers \& Education, 56(4), 1023-1031. https://doi.org/10.1016/j.compedu.2010.12.002

İBìLİ, E. (2019). The Use of Dynamic Geometry Software from a Pedagogical Perspective: Current Status and Future Prospects. Journal of Computer and Education Research, 714), 337-355. https://doi.org/10.18009/jcer.579517

Ivankova, N. V, Creswell, J. W., \& Stick, S. L. (2006). Using mixed-methods sequential explanatory design: From theory to practice. Field Methods, 18(1), 3-20. https://doi.org/10.1177/1525822X05282260

Jeon, Y.-J. (2015). A study on technology embedded english classes using qr codes. International Journal of Contents, 11(1), 1-6. https://doi.org/10.5392/IJoC.2015.11.1.001

Keller, J. M. (2016). Motivation, learning, and technology: Applying the ARCS-V motivation model. Participatory Educational Research, 3(2), 1-15. https://doi.org/10.17275/per.16.06.3.2

Kesharwani, A. (2020). Do (how) digital natives adopt a new technology differently than digital immigrants? A longitudinal study. Information \& Management, 57(2), 103170. https://doi.org/10.1016/j.im.2019.103170

Kolář, P. (2019). GeoGebra for Secondary School Physics. Journal of Physics: Conference Series, 1223(1), 12008. https://doi.org/10.1088/1742-6596/1223/1/012008

König, A., \& Bernsen, D. (2014). Mobile learning in history education. Journal of Educational Media, Memory, and Society, 6(1), 107-123. https://doi.org/10.3167/jemms.2014.060106

Kösa, T., \& Karakuş, F. (2010). Using dynamic geometry software Cabri 3D for teaching analytic geometry. Procedia-Social and Behavioral Sciences, 2(2), 1385-1389. https://doi.org/10.1016/j.sbspro.2010.03.204

Lan, Y.-F., Tsai, P.-W., Yang, S.-H., \& Hung, C.-L. (2012). Comparing the social knowledge construction behavioral patterns of problem-based online asynchronous discussion in e/m-learning environments. Computers \& Education, 59(4), 1122-1135. https://doi.org/10.1016/j.compedu.2012.05.004

Ljajko, E., \& Ibro, V. (2013). Development of Ideas in a GeoGebra-Aided Mathematics Instruction. Online Submission, 3(3), 1-7. https://doi.org/10.13054/mije.si.2013.01

Motiwalla, L. F. (2007). Mobile learning: A framework and evaluation. Computers \& Education, 49(3), 581-596. https://doi.org/10.1016/j.compedu.2005.10.011 
Mudaly, V., \& Fletcher, T. (2019). The effectiveness of Geogebra when teaching linear functions using the iPad. Problems of Education in the 21st Century, 771), 55-81. https://doi.org/0.33225/pec/19.77.55

Pachler, N., Bachmair, B., \& Cook, J. (2010a). Mobile learning: A topography. In Mobile Learning, 29-72. https://doi.org/10.1007/978-1-4419-0585-7_2

Pachler, N., Bachmair, B., \& Cook, J. (2010b). Mobile Learning: Structures, Agency, Practices. https://doi.org/10.1007/978-1-4419-0585-7

Pamungkas, M. D., Rahmawati, F., \& Dinara, H. A. (2020). Integrating GeoGebra into Space Geometry in College. 3rd International Conference on Learning Innovation and Quality Education (ICLIQE 2019), 999-1006. https://doi.org/10.2991/assehr.k.200129.123

Pilli, O., \& Aksu, M. (2013). The effects of computer-assisted instruction on the achievement, attitudes and retention of fourth grade mathematics students in North Cyprus. $\begin{array}{llll}\text { Computers } \& \text { Education, 62), } & \text { 62 }\end{array}$ https://doi.org/10.1016/j.compedu.2012.10.010

Pimmer, C., Mateescu, M., \& Gröhbiel, U. (2016). Mobile and ubiquitous learning in higher education settings. A systematic review of empirical studies. Computers in Human Behavior, (63), 490-501. https://doi.org/10.1016/j.chb.2016.05.057

Radakovic, N., \& McDougall, D. (2012). Using dynamic geometry software for teaching conditional probability with area-proportional Venn diagrams. International Journal of Mathematical Education in Science and Technology, 43(7), 949-953. https://doi.org/10.1080/0020739X.2011.633628

Reisa, Z. A. (2010). Computer supported mathematics with Geogebra. Procedia-Social and Behavioral Sciences, (9), 1449-1455. https://doi.org/10.1016/j.sbspro.2010.12.348

Rybanska, V., McKay, R., Jong, J., \& Whitehouse, H. (2018). Rituals improve children's ability to delay gratification. Child Development, 89(2), 349-359. https://doi.org/10.1111/cdev.12762

Schuck, S., Kearney, M., \& Burden, K. (2017). Exploring mobile learning in the Third Space. Technology, Pedagogy and Education, 26(2), 121-137. https://doi.org/10.1080/1475939X.2016.1230555

Solomon, R. L. (1949). An extension of control group design. Psychological Bulletin, 46(2), 137. https://doi.org/10.1037/h0062958

Sung, Y.-T., Chang, K.-E., \& Liu, T.-C. (2016). The effects of integrating mobile devices with teaching and learning on students' learning performance: A meta-analysis and 
research synthesis. Computers \& Education, (94), 252-275. https://doi.org/10.1016/j.compedu.2015.11.008

Tomaschko, M., \& Hohenwarter, M. (2018). Usability Evaluation of a Mobile Graphing Calculator Application Using Eye Tracking. International Conference on Learning and Collaboration Technologies, 180-190. https://doi.org/10.1007/978-3-319-917436_14

Van Landschoot, R., Portzky, G., \& Van Heeringen, K. (2017). Knowledge, self-confidence and attitudes towards suicidal patients at emergency and psychiatric departments: A randomised controlled trial of the effects of an educational poster campaign. International Journal of Environmental Research and Public Health, 14(3), 304. https://doi.org/10.3390/ijerph14030304

Vanden Abeele, M. M. P. (2016). Mobile youth culture: A conceptual development. Mobile Media \& Communication, 4(1), 85-101. https://doi.org/10.1177/2050157915601455

Wu, W.-H., Wu, Y.-C. J., Chen, C.-Y., Kao, H.-Y., Lin, C.-H., \& Huang, S.-H. (2012). Review of trends from mobile learning studies: A meta-analysis. Computers \& Education, 59(2), 817-827. https://doi.org/10.1016/j.compedu.2012.03.016

Zazkis, R. (2020). Technology in Mathematics Teacher Education on Trust and Pitfalls. In STEM Teachers and Teaching in the Digital Era (pp. 243-259). https://doi.org/10.1007/978-3-030-29396-3_13

Zengin, Y., Furkan, H., \& Kutluca, T. (2012). The effect of dynamic mathematics software geogebra on student achievement in teaching of trigonometry. Procedia-Social and Behavioral Sciences, (31), 183-187. https://doi.org/10.1016/j.sbspro.2011.12.038

Zulnaidi, H., Oktavika, E., \& Hidayat, R. (2020). Effect of use of GeoGebra on achievement of high school mathematics students. Education and Information Technologies, 25(1), 51-72. https://doi.org/10.1007/s10639-019-09899-y 